\title{
Dacryocystography Using Cone Beam CT in Patients With Lacrimal Drainage System Obstruction
}

\author{
Markus Tschopp, M.D.*†, Michael M. Bornstein, D.M.D.ł\$, Pedram Sendi, D.M.D., M.D. //, \\ Reinhilde Jacobs, D.D.S., Ph.D.§, and David Goldblum, M.D.* \\ *Department of Ophthalmology, University Basel, University Hospital Basel, Basel; †Department of \\ Ophthalmology, University Bern, Inselspital, Bern; $\neq$ Department of Oral Surgery and Stomatology, \\ School of Dental Medicine, University of Bern, Bern, Switzerland; §OMFS IMPATH research group, \\ Department of Imaging and Pathology, Faculty of Medicine, University of Leuven, Leuven, Belgium; and \\ //Institute for Clinical Epidemiology \& Biostatistics, University Hospital Basel, Basel, Switzerland
}

Purpose: To assess the usefulness of cone beam CT (CBCT) for dacryocystography (DCG) using either direct syringing or passive application of contrast medium.

Methods: Ten consecutive patients with epiphora who had CBCT-DCG in a sitting position were retrospectively analyzed. CBCT-DCGs were performed using 2 techniques: direct syringing with contrast medium or using the passive technique, where patients received 3 drops of contrast medium into the conjunctival sac before CBCT-DCG. Clinical and radiologic diagnoses were compared for both groups.

Results: The 10 patients $($ men $=3)$ had a mean age of 63.2 years. Both techniques proved to be simple procedures with good delineation of the bone, soft tissue, and the contrast medium in the lacrimal system. No side effects were noted.

Conclusions: CBCT-DCG is a useful alternative to determine the localization of stenosis in patients with chronic epiphora.

(Ophthal Plast Reconstr Surg 2014;30:486-491)

$\mathrm{D}$ acryocystography (DCG) was the first radiologic technique to visualize the nasolacrimal system in patients with epiphora. ${ }^{1}$ With ongoing technological development, $\mathrm{CT}^{2,3}$ and MRI have been adapted for DCG. Disadvantages reported with CT-DCG are the lack of a dynamic contrast passage and increased radiation exposure. ${ }^{4}$ MRI-DCG can show the function of the nasolacrimal apparatus under physiologic conditions after topical application of a contrast medium and visualizes subtle soft-tissue changes, such as early malignancies. ${ }^{5}$ Long imaging time, poor delineation of bony ductal components, and lack of anatomical detail has prevented this imaging technique from being routinely used. Both the abovementioned techniques also have the drawback of being performed only in the supine position. The present investigation presents 10 patients diagnosed with epiphora who were evaluated by DCG using cone beam CT (CBCT) with 2 different methods of contrast medium application (direct syringing versus passive drainage of contrast medium) prior to surgical intervention. The primary objective of

Accepted for publication January 23, 2014.

The authors have no financial or conflicts of interest to disclose

Address correspondence and reprint requests to David Goldblum, M.D., Department of Ophthalmology, University Basel and University Hospital Basel, Mittlere Strasse 91, 4031 Basel, Switzerland. E-mail: david. goldblum@usb.ch

DOI: 10.1097/IOP.0000000000000154 the present study was to evaluate CBCT-DCG as an alternative to conventional and CT-based DCG and to compare CBCT-DCG findings to clinical findings on syringing of the lacrimal system.

\section{PATIENTS AND METHODS}

Patient Selection. This study comprised 10 consecutive patients referred to the university eye clinic Bern in whom a DCG was necessary: 9 patients with unilateral and 1 patient with bilateral obstruction of the nasolacrimal drainage system. All patients had undergone prior clinical examination by 1 experienced ophthalmologist (M.T.) on the basis of symptoms of unilateral or bilateral epiphora, suggesting unilateral or bilateral obstruction of the nasolacrimal drainage system. The region of obstruction was assessed clinically by syringing of the nasolacrimal system. A Bangerter lacrimal cannula was passed into the punctum and advanced to the medial wall of the lacrimal sac fossa. Irrigation with saline solution ensured a stenosis, and only patients reporting the absence of saline solution in their nasal antrum and/or pharynx (implying an obstruction of the lacrimal drainage system) were included in the study. If the cannula reached bone (hard stop), the obstruction was classified as postsaccal; if the cannula did not reach bone (soft stop), the obstruction was classified as canalicular. Informed consent was obtained from all patients for further imaging using CBCT. Subjects presenting with iodine or contrast material solution allergies were excluded. The ophthalmologist referred patients for DCG using 2 different techniques for contrast medium application. The first patients were examined with the direct syringing method, after which the passive method was applied. The first 5 patients examined with each DCG method were selected and analyzed. Examinations and data collection were performed according to guidelines of the World Medical Association Declaration of Helsinki and the ethical committee of the state of Bern.

CBCT Settings. The CBCT images were obtained with a 3-dimensional (3D) Accuitomo 170 XYZ Slice View Tomograph (Morita, Kyoto, Japan). Operating parameters were set at 5.0 to $7.0 \mathrm{~mA}$ and $80 \mathrm{kV}$, and exposure time was 17.5 seconds. For all CBCT images, a limited field of view (FOV) of $6 \times 5 \mathrm{~cm}$ or $8 \times 5 \mathrm{~cm}$ was selected. The data were reconstructed with slices of $0.5-\mathrm{mm}$ thickness at $0.5-\mathrm{mm}$ intervals. The analyses described below were performed using a specialized computer software (I-View, Version 2.0.4.5; Morita, Kyoto, Japan).

DCG Procedures. For the direct syringing technique, all patients initially received 1 drop topical anesthetic (oxybuprocain $0.4 \%$, Novesin; OmniVision AG, Neuhausen, Switzerland) into the conjunctival sac of OU. Thereafter, the inferior punctum of OU was catheterized with a 26-G microcatheter (Vasculon Plus; Becton Dickinson AG, Allschwil, Switzerland), and 1 to $2 \mathrm{ml}$ of a nonionic, water-soluble contrast medium (Iopamiro 300; Bracco Suisse SA, Manno, Switzerland) was slowly 
TABLE 1. Clinical and radiologic parameters of the 5 patients with epiphora who underwent cone beam CT-based dacryocystography using a direct syringing technique (patient 5 had bilateral obstruction)

\begin{tabular}{|c|c|c|c|c|c|}
\hline Patient & Gender & Age & Clinical level of obstruction & Side & Radiographic level of obstruction \\
\hline 1 & Female & 71 & Postsaccal & Right & Nasolacrimal duct \\
\hline 2 & Female & 69.5 & Postsaccal & Left & Nasolacrimal duct \\
\hline 3 & Male & 61.5 & Postsaccal & Right & Canalicular \\
\hline 4 & Female & 51 & Postsaccal & Left & Nasolacrimal duct \\
\hline 5 & Female & 64 & Postsaccal & Right & Canalicular \\
\hline 5 & Female & 64 & Postsaccal & Left & Lacrimal sac \\
\hline
\end{tabular}

TABLE 2. Clinical and radiologic parameters of the 5 patients with epiphora who underwent cone beam CT-based dacryocystography using a passive technique of contrast medium application

\begin{tabular}{lccccc}
\hline Patient & Gender & Age & Clinical level of obstruction & Side & Radiographic level of obstruction \\
\hline 6 & Female & 37 & Canalicular & Left & Lacrimal sac \\
7 & Female & 71 & Canalicular & Right & Canalicular \\
8 & Male & 66 & Postsaccal & Right & Nasolacrimal duct \\
9 & Female & 44 & Postsaccal & Left & Nasolacrimal duct \\
10 & Male & 45 & Canalicular & Canalicular \\
\hline
\end{tabular}
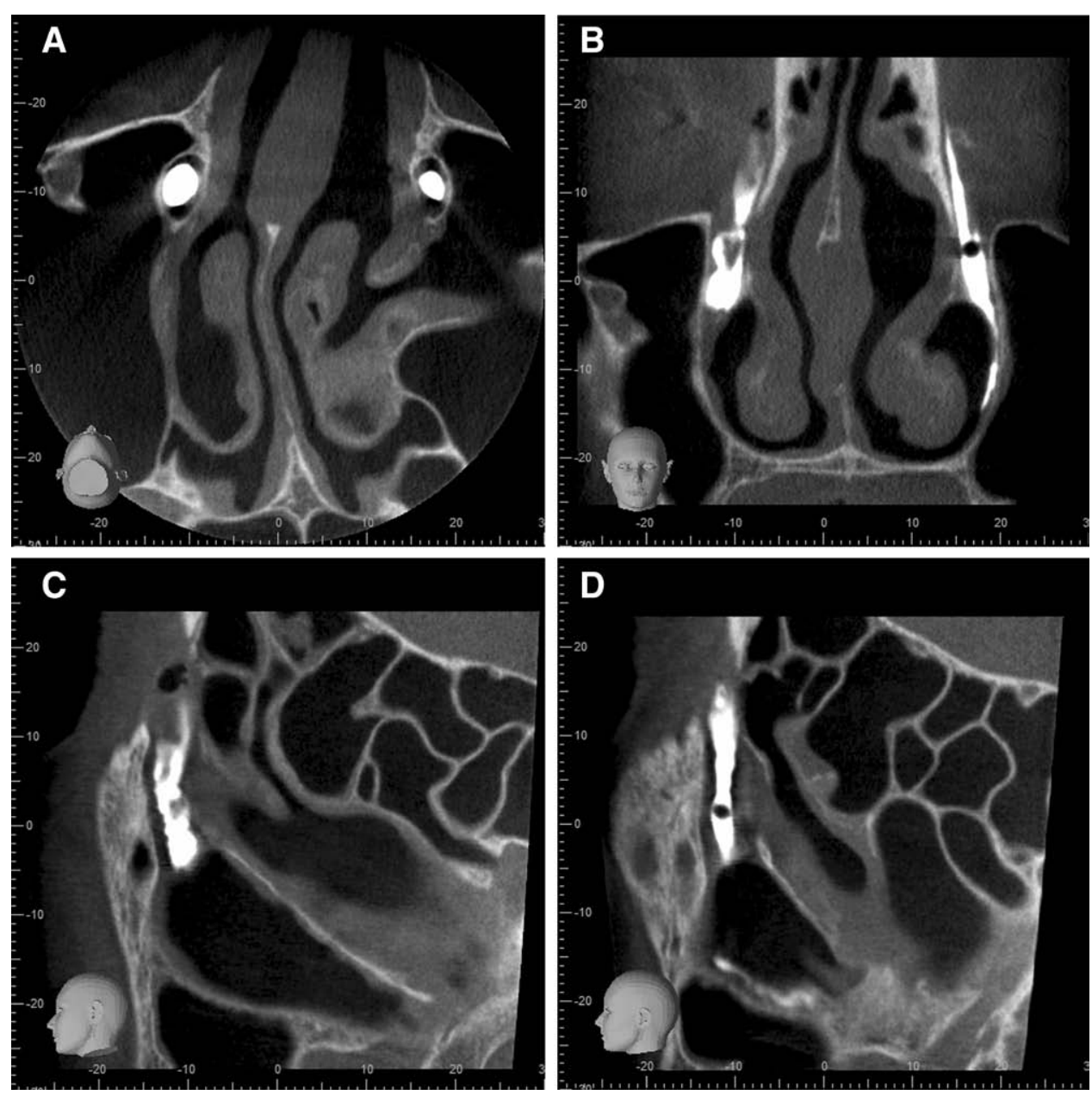

FIG. 1. Dacryocystography with direct application of contrast medium using cone beam CT in a 71-year-old female patient with the obstruction of the right nasolacrimal duct near the exit at the lower nasal meatus. A, In the axial slice, there is more contrast medium visible in the right nasolacrimal duct, indicating a blockage. B, In the coronal slice, the contrast medium is running through the whole left nasolacrimal system, with traces of the medium in the nasal antrum. C, Sagittal slice of the obstructed right nasolacrimal system. D, Sagittal slice of the patent left nasolacrimal system. 
injected manually until the patients reported either feeling the solution in their nasal antrum and/or pharynx or when the liquid refluxed out of the lower or upper punctum.

For the passive technique, the patients received a total of 3 drops of Iopamiro 300 into the conjunctival sac of OU after 0,5 , and 10 minutes, and they were told not to rub their eyes. Thereafter, CBCT was immediately performed.

Image Analysis. CBCT images were analyzed by an experienced dentomaxillofacial radiologist (M.M.B.) and an ophthalmologist (M.T.). An obstruction was considered as present when no contrast medium was visible (as a thin hyperdense film or radio-opaque islands) in the nasal antrum, suggesting a blockage of the respective nasolacrimal system. The location of obstruction was classified into the region of the lacrimal canaliculi, the lacrimal sac, or the lacrimal duct according to anatomical landmarks of the distribution of the contrast medium. Radiologic findings were related to clinical findings for both groups. Furthermore, the diagnostic results obtained with both methods were compared, whereas in patients with diseased versus healthy sides, the differences were studied and radiologic findings described.

StatisticalAnalysis. Data were first analyzed descriptively. Between-group differences of categorical variables were compared using the chi-square test and continuous variables (i.e., age) using the nonparametric Wilcoxon rank-sum test. Agreement between measurement techniques was estimated using Cohen kappa. All statistical analyses were performed using Stata 11.2 (StataCorp, College Station, TX, U.S.A.).

\section{RESULTS}

A total of 10 patients with epiphora referred for further diagnostics using CBCT were included in the present analysis. They included 3 men and had a mean age of 63.2 years. For each DCG technique (direct syringing and passive), the first 5 patients seen at the Section of Dental Radiology and Stomatology were selected (Tables 1 and 2). There was no statistically significant difference between both groups regarding age $(p=0.295)$, gender $(p=0.424)$, and the side of obstruction of the nasolacrimal system $(p=0.608)$.

Feasibility of CBCT. No side effects were noted during catheterization or application of the contrast medium in both methods. Although the application of anesthetic eye drops and the syringing was regarded as slightly unpleasant by some patients, the contrast medium did not cause any irritation. Overall, both methods were well tolerated by all patients. The image acquisition time was 17.5 seconds for all patients, and the whole procedure was performed in less than 15 minutes (including application of contrast medium). Both techniques exhibited good delineation of bony structures, soft tissue, and the contrast medium in the lacrimal system.
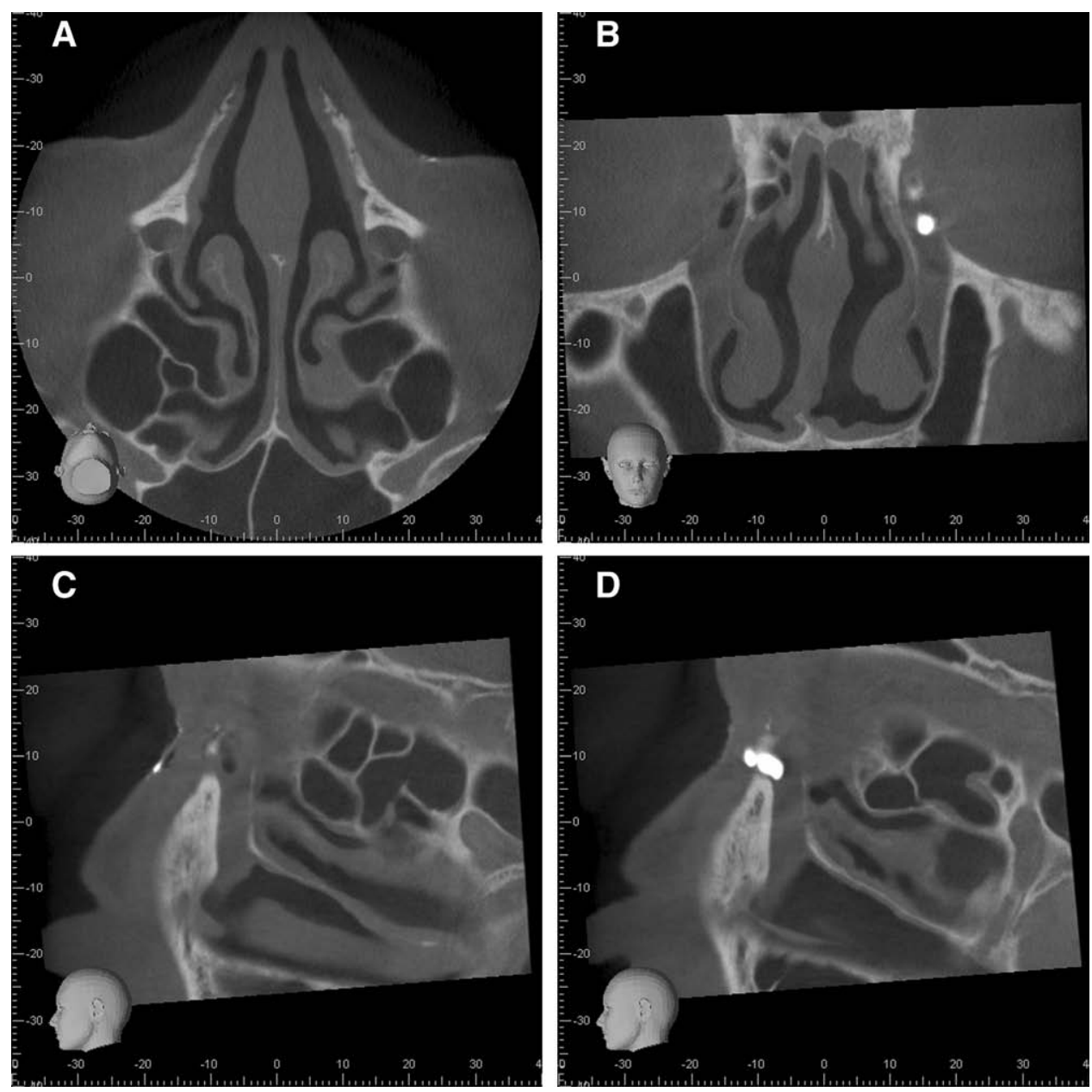

FIG. 2. Dacryocystography with direct application of contrast medium using cone beam CT in a 64-year-old female patient with bilateral obstruction of the nasolacrimal system. A, In the axial slice, no contrast medium is visible, indicating the blockage of both nasolacrimal ducts at the canalicular level. B, In the coronal slice, the contrast medium is visible as being pooled in the left lacrimal sac. C, Sagittal slice of the right nasolacrimal system exhibiting contrast medium in the common nasolacrimal canaliculus. D, Sagittal slice of the left nasolacrimal system showing blockage at the lacrimal sac. 
Descriptive Analysis of the CBCT Findings in the Direct Syringing Group. In this group, a total of 6 nasolacrimal systems were found to be radiologically obstructed in 5 patients ( 1 patient had bilateral obstruction). In 2 patients the obstruction was at the canalicular level and in 3 patients in the course of the nasolacrimal duct. Only in 1 case, the lacrimal sac was diagnosed as the site of obstruction. A detailed analysis of the radiologic findings is provided in Table 1 and Figures 1 and 2 .

Descriptive Analysis of the CBCT Findings in the Passive Application Group. In this group, 5 nasolacrimal systems were found to be radiologically obstructed in 5 patients; 2 at the canalicular level and 2 in the course of the nasolacrimal duct. Similar to the direct syringing group, obstruction at the level of the lacrimal sac was seen in only 1 patient. A detailed analysis of the radiologic findings is provided in Table 2 and Figures 3 and 4 .

Correlation Between Clinical and Radiologic Findings Using the 2 DCG Techniques. All clinically diagnosed obstructions were detected with CBCT-DCG $(\mathrm{n}=11)$. All clinical patent lacrimal systems were correctly classified as patent with radiography $(n=9)$. The overall correlation between the clinical (canalicular or postsaccal) and radiologic levels of obstruction (canalicular, lacrimal sac, or nasolacrimal duct) for both CBCT techniques used was $72.7 \%$ (as clinically it is not possible to distinguish between an obstruction in the lacrimal sac or nasolacrimal duct, these 2 levels were combined for the analysis). This resulted in a Cohen kappa coefficient of 0.38 for both groups, representing a fair correlation. For the direct syringing technique, the correlation was $66.7 \%$ and the respective Cohen kappa value 0.00 , indicating a weak correlation. For the passive technique, the correlation was $80 \%$ and the Cohen kappa value 0.62 , indicating a good correlation.

\section{DISCUSSION}

In the present study including 10 patients with epiphora due to obstruction of the nasolacrimal system, the site of resistance was found to be 5 times at the level of the nasolacrimal duct, 4 times at the level of the canaliculi or common canaliculus, and twice at the level of the lacrimal sac. Because of the low sample size, comparisons with reported data in the literature were difficult. Moreover, differences and even controversies regarding the exact incidence rates of the location of obstruction in the nasolacrimal system exist in the literature. ${ }^{6}$

With advancing age, epiphora becomes more frequent because of progressive fibrosis resulting from chronic inflammation leading to stenosis, and it has been more commonly found
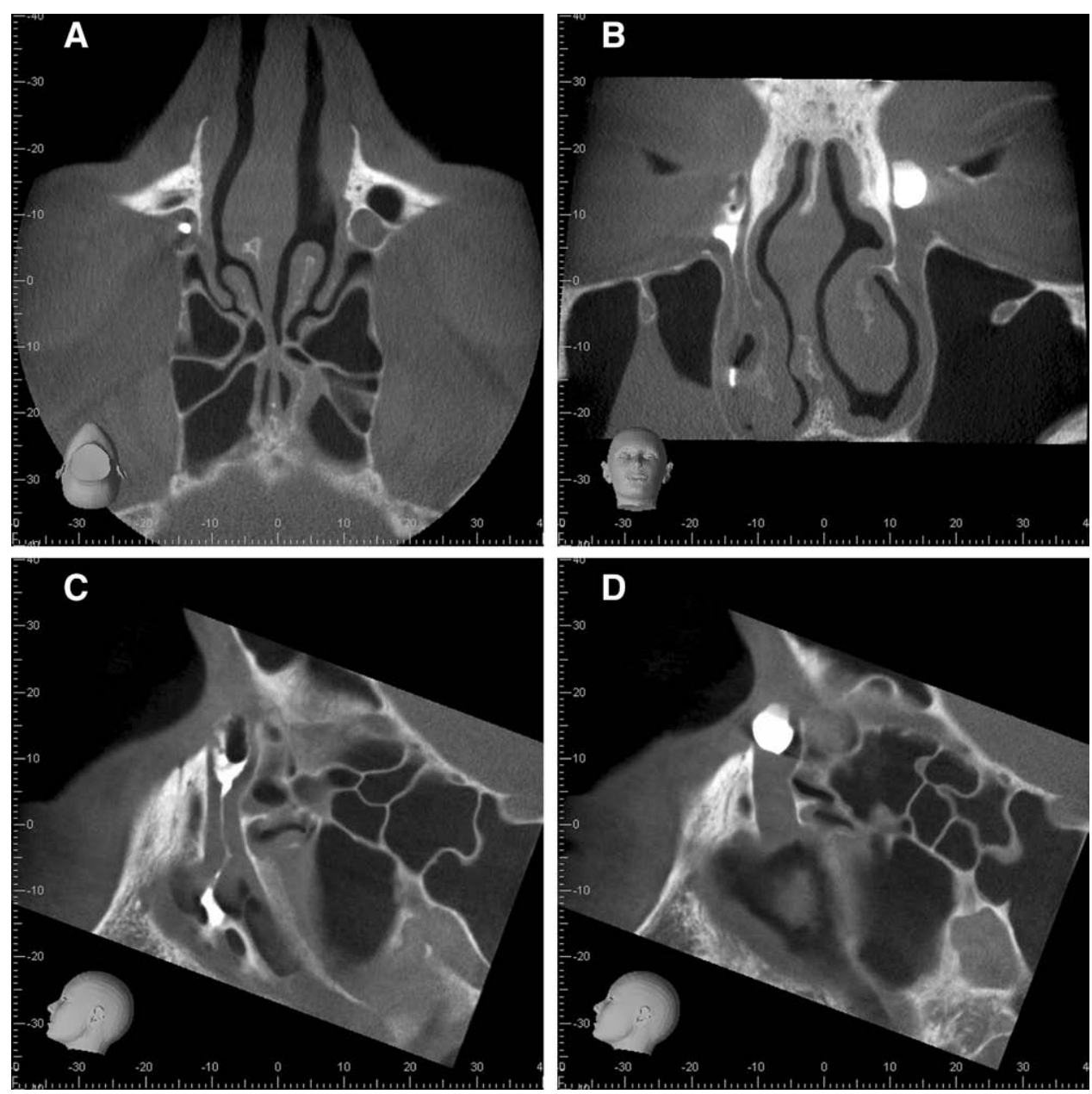

FIG. 3. Dacryocystography after passive application of contrast medium using cone beam CT in a 37-year-old female patient with the obstruction of the left nasolacrimal system at the level of the lacrimal sac. A, In the axial slice, no contrast medium is visible in the left nasolacrimal duct, indicating a blockage at the distal end of the lacrimal sac. B, In the coronal slice, the contrast medium is observed through the whole right nasolacrimal system, with traces in the nasal antrum, whereas on the left site, pooling of the contrast agent is visible at the lacrimal sac. C, Sagittal slice of the right nasolacrimal system. D, Sagittal slice of the left nasolacrimal system showing blockage at the lacrimal sac. 

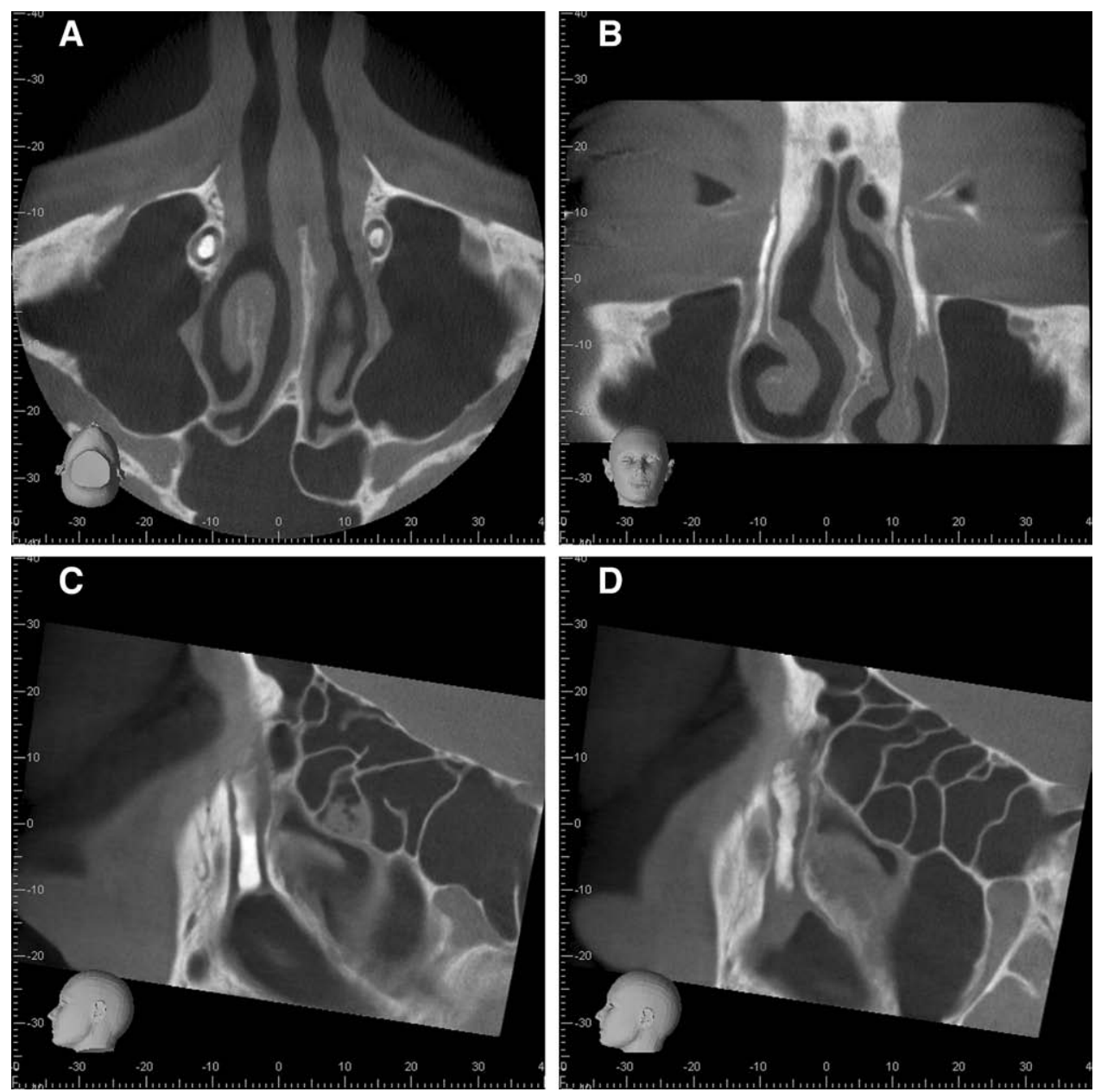

FIG. 4. Dacryocystography after passive application of contrast medium using cone beam CT in a 44-year-old female patient with obstruction of the left nasolacrimal duct. A, In the axial slice, the contrast medium visible in both nasolacrimal ducts. B, In the coronal slice, the contrast medium is observed through the whole right nasolacrimal system, with traces in the nasal antrum itself, whereas on the left site, it is blocked in the intraosseous portion of the nasolacrimal duct. C, Sagittal slice of the right nasolacrimal system. D, Sagittal slice of the left nasolacrimal system.

in women. ${ }^{7,8}$ In this study, $70 \%$ of the patients were women, and the overall average age was 63.2 years. Burning sensation, irritation, and eye dryness has been reported as side effects after the instillation of iodinated water-soluble contrast medium. ${ }^{9}$ In the present series of 10 patients, no adverse side effects were registered, and DCG was performed as planned in all patients. Regarding the 2 different techniques used - direct syringing and passive technique - the exact location with classic radiologic features such as prestenotic enlargement due to increased contrast medium injection could be diagnosed and seen. ${ }^{4}$

The drainage of tears is actively supported by a physiologic pumping mechanism that occurs with the closure and opening of eyes. ${ }^{10}$ Potential causes of epiphora associated with impaired tear outflow may be functional, structural, or both. ${ }^{11}$ With the direct syringing technique, the contrast material is introduced manually with some pressure into the canaliculi; subsequently, the visualization of the lacrimal outflow is not considered physiologic, and hence functional tearing may not be found. ${ }^{12}$ With the passive technique, the contrast material is administered into the lower conjunctival sac in a physiologic way using 3 drops of the contrast medium. As CBCT is performed in an upright position, the lacrimal outflow is established in a physiologic way. Thus, the authors expect that functional epiphora could be detected with the passive technique, which may offer an alternative to dacryoscintigraphy. Although not performed in this study, syringing of the lacrimal system with contrast medium after image acquisition with the passive method may open functional obstructions and discriminate between functional and structural obstruction. Depending on the clinicians needs, direct syringing can be carried out to detect structural obstruction, and the passive method can be used to detect functional obstruction; in addition, a combination of the 2 methods can be used. To the best of the authors' knowledge, only 1 study has described CBCT-DCG in the literature. ${ }^{4}$ That study applied an approach similar to the direct syringing method in the present study. In that study, of 45 patients, 8 patients showed a normal filling with contrast medium despite severe epiphora. It remains an intriguing question whether the passive method might have diagnosed a functional stenosis in some of these patients.

In the present study, there was a marked difference regarding the Cohen kappa values, with direct syringing exhibiting a weak correlation and the passive technique having a very good correlation between clinical and radiologic diagnosis of the site of obstruction. These data have to be interpreted with some caution because of the limited sample size, and further research with larger groups in a prospective randomized design should be conducted. 
High-resolution thin-section CT has been reported to be an appropriate and useful modality for $3 \mathrm{D}$ assessment of the nasolacrimal drainage system, its surrounding bony structures, adjacent regions of the orbit, facial skeleton, paranasal sinuses, and the nasal cavity. ${ }^{13}$ One major disadvantage encountered when using $\mathrm{CT}$ is the exposure of radiosensitive ocular structures to radiation. This should be avoided whenever possible because the lens, retina, and optic nerve are among the tissues in the head and neck region that are most sensitive to ionizing radiation. ${ }^{14}$ MRI-DCG has the major advantage that it prevents the exposure of high-dose ionizing radiation on the lens, retina, and optic nerve..$^{15,16}$ However, because of the long imaging time and poor delineation of bony ductal components, it is not used on a routine basis. Since its first description in $1998,{ }^{17}$ CBCT has become a popular and valuable technique for preoperative diagnosis and various dental indications including dental and maxillofacial traumatology. ${ }^{18-27}$ In a recent study measuring radiation doses of DCG performed using CBCT and multislice $\mathrm{CT}$, the radiation dose levels in $\mathrm{mSv}$ to lens, parotid gland, and thyroid gland were substantially less for CBCT. ${ }^{4}$ For dental multislice CT exposures, effective doses up to $1 \mathrm{mSv}$ are used, but considerably lower doses are used for single jaw or low-dose protocols. ${ }^{28} \mathrm{~A}$ recent review article evaluating effective doses for dental $\mathrm{x}$-ray imaging presented a dose range of 0.019 to $0.674 \mathrm{mSv}$ for dentoalveolar (small to medium) FOVs and 0.030 to $1.073 \mathrm{mSv}$ for a craniofacial (large) FOV compared with a dose range of 0.280 to $1.410 \mathrm{mSv}$ for CT modalities. ${ }^{26}$ In the present study, a small to medium FOV protocol was applied for CBCT-DCG $(6 \times 5 \mathrm{~cm}$ or $8 \times 5 \mathrm{~cm})$. The average dose for all CBCT devices in the market was found to be $88 \mu \mathrm{Sv}$ and $34 \mu \mathrm{Sv}$, respectively, compared with $131 \mu \mathrm{Sv}$ for large FOVs. ${ }^{29}$ On the basis of these findings, the authors believe that $\mathrm{CBCT}$ results in radiation doses well below those reported for common multislice CT protocols and that CBCT offers the advantage to perform imaging by an appropriate selection of exposure parameters and field size, depending on the diagnostic requirements.

In conclusion, the present investigation demonstrated that CBCT-DCG has potential for evaluating patients with epiphora and a tentative diagnosis of complete obstruction of the nasolacrimal system prior to surgical intervention. Advantages of the CBCT-DCG include short image acquisition time and good delineation of bone and soft tissue at a lower irradiation dose than conventional CT. Furthermore, CBCT-DCG is performed in an upright position, allowing physiologic examination of the lacrimal system.

\section{REFERENCES}

1. Conway ST. Evaluation and management of "functional" nasolacrimal blockage: results of a survey of the American Society of Ophthalmic Plastic and Reconstructive surgery. Ophthal Plast Reconstr Surg 1994;10:185-7; discussion 188.

2. Francis IC, Kappagoda MB, Cole IE, et al. Computed tomography of the lacrimal drainage system: retrospective study of 107 cases of dacryostenosis. Ophthal Plast Reconstr Surg 1999;15:217-26.

3. Bonnet F, Ducasse A, Marcus C, et al. CT dacryocystography: normal findings and pathology. J Radiol 2009;90(11 pt 1):1685-93.

4. Wilhelm KE, Rudorf $\mathrm{H}$, Greschus S, et al. Cone-beam computed tomography (CBCT) dacryocystography for imaging of the nasolacrimal duct system. Klin Neuroradiol 2009;19:283-91.

5. Wilhelm K, Keller E, Textor J, et al. MRI dacryocystography after applying gadolinium-containing eyedrops. Rofo 1997;167:58-61.

6. Mandeville JT, Woog JJ. Obstruction of the lacrimal drainage system. Curr Opin Ophthalmol 2002;13:303-9.

7. Schaumberg DA, Sullivan DA, Buring JE, et al. Prevalence of dry eye syndrome among US women. Am J Ophthalmol 2003;136:318-26.
8. Schaumberg DA, Dana R, Buring JE, et al. Prevalence of dry eye disease among US men: estimates from the Physicians' Health Studies. Arch Ophthalmol 2009;127:763-8.

9. Caldemeyer KS, Stockberger SM Jr, Broderick LS. Topical contrast-enhanced CT and MR dacryocystography: imaging the lacrimal drainage apparatus of healthy volunteers. AJR Am J Roentgenol 1998;171:1501-4.

10. Lee MJ, Kyung HS, Han MH, et al. Evaluation of lacrimal tear drainage mechanism using dynamic fluoroscopic dacryocystography. Ophthal Plast Reconstr Surg 2011;27:164-7.

11. Freitag SK, Woog JJ, Kousoubris PD, et al. Helical computed tomographic dacryocystography with three-dimensional reconstruction: a new view of the lacrimal drainage system. Ophthal Plast Reconstr Surg 2002;18:121-32.

12. Blackmore KJ, Ainsworth G, Robson AK. Epiphora: an evidence based approach to the 12 minute consultation. Clin Otolaryngol 2010;35:210-4

13. Glatt HJ, Chan AC, Barret L. Computed tomography of nasolacrimal duct obstruction after endoscopic sinus surgery. Arch Otolaryngol Head Neck Surg 1991;117:1059-60.

14. Cubuk R, Tasali N, Aydin S, et al. Dynamic MR dacryocystography in patients with epiphora. Eur J Radiol 2010;73:230-3.

15. Rubin PA, Bilyk JR, Shore JW, et al. Magnetic resonance imaging of the lacrimal drainage system. Ophthalmology 1994;101:235-43.

16. Conneely MF, Hacein-Bey L, Jay WM. Magnetic resonance imaging of the orbit. Semin Ophthalmol 2008;23:179-89.

17. Mozzo P, Procacci C, Tacconi A, et al. A new volumetric CT machine for dental imaging based on the cone-beam technique: preliminary results. Eur Radiol 1998;8:1558-64.

18. De Vos W, Casselman J, Swennen GR. Cone-beam computerized tomography (CBCT) imaging of the oral and maxillofacial region: a systematic review of the literature. Int J Oral Maxillofac Surg 2009;38:609-25.

19. Patel S. New dimensions in endodontic imaging: Part 2. Cone beam computed tomography. Int Endod J 2009;42:463-75.

20. Jeger FB, Janner SF, Bornstein MM, et al. Endodontic working length measurement with preexisting cone-beam computed tomography scanning: a prospective, controlled clinical study. $J$ Endod 2012;38:884-8

21. Bornstein MM, Wölner-Hanssen AB, Sendi P, et al. Comparison of intraoral radiography and limited cone beam computed tomography for the assessment of root-fractured permanent teeth. Dent Traumatol 2009;25:571-7.

22. Stuck BA, Hülse R, Barth TJ. Intraoperative cone beam computed tomography in the management of facial fractures. Int J Oral Maxillofac Surg 2012;41:1171-5.

23. Low KM, Dula K, Bürgin W, et al. Comparison of periapical radiography and limited cone-beam tomography in posterior maxillary teeth referred for apical surgery. J Endod 2008;34:557-62.

24. Bornstein MM, Lauber R, Sendi P, et al. Comparison of periapical radiography and limited cone-beam computed tomography in mandibular molars for analysis of anatomical landmarks before apical surgery. J Endod 2011;37:151-7

25. Walter C, Kaner D, Berndt DC, et al. Three-dimensional imaging as a pre-operative tool in decision making for furcation surgery. $J$ Clin Periodontol 2009;36:250-7.

26. Harris D, Horner K, Gröndahl K, et al. E.A.O. guidelines for the use of diagnostic imaging in implant dentistry 2011. A consensus workshop organized by the European Association for Osseointegration at the Medical University of Warsaw. Clin Oral Implants Res 2012;23:1243-53.

27. Bornstein MM, Scarfe WC, Vaughn VM, Jacobs R: Cone beam computed tomography in implant dentistry: a systematic review focusing on guidelines, indications, and radiation dose risks. Int $J$ Oral Maxillofac Implants. 2014;29 (Suppl):55-77.

28. Loubele M, Bogaerts R, Van Dijck E, et al. Comparison between effective radiation dose of CBCT and MSCT scanners for dentomaxillofacial applications. Eur J Radiol 2009;71:461-8.

29. Pauwels R, Beinsberger J, Collaert B, et al.; SEDENTEXCT Project Consortium. Effective dose range for dental cone beam computed tomography scanners. Eur J Radiol 2012;81:267-71. 